Supporting File 1. Study Skills Intervention Slides

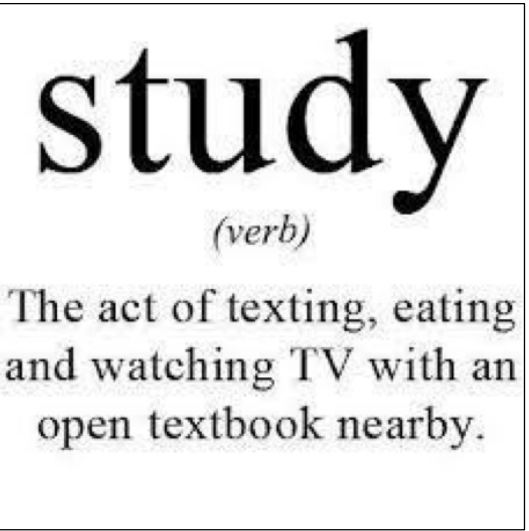

What are you doing?

When are you doing it?

\title{
What are you doing?
}

- Discuss with your neighbor:

- How you have been preparing for the class so far?

- How do you typically prepare for exams? 


\section{What are you doing?}

- Discuss with your neighbor:

- How you have been preparing for the class so far?

- How do you typically prepare for exams?

Rank these studying methods from least useful to most useful in terms of how much you will retain of what you learned A - reading a textbook $B$ - answering questions (self-testing) $\mathrm{C}$ - listening to a lecture D - discussing with your classmates

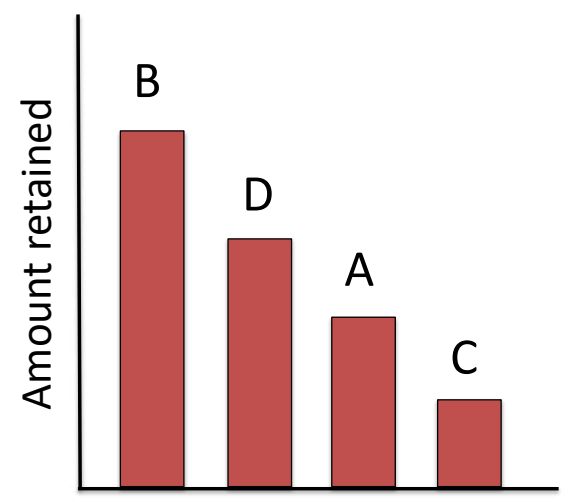

Self-testing is key! 


\section{The Learning Pyramid*}

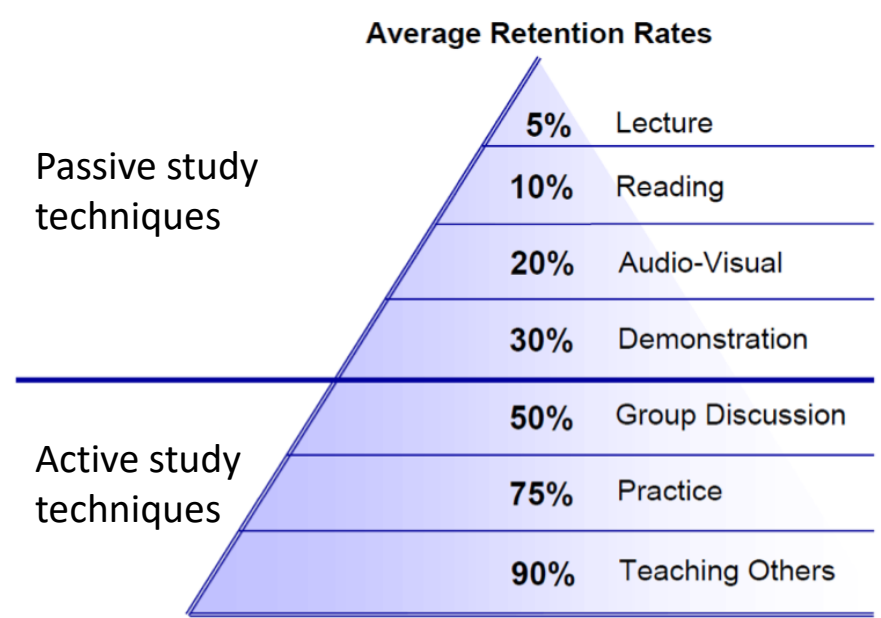

*Adapted from National Training Laboratories. Bethel, Maine

\section{Effective Self-Testing Techniques}

- Answering practice questions

- Recalling course concepts from memory

- Explaining course concepts to classmates (or yourself)

- Self-testing reveals what you truly know and what you truly don't 


\section{When are you doing it?}

- How do you typically prepare for a quiz?

\section{When are you doing it?}

\begin{tabular}{||l|l|l|l|l|l|l||}
\hline \multicolumn{7}{|c|}{2016 APRIL } \\
\hline SUN & MON & TUE & WED & THU & \multicolumn{1}{|c|}{ FRI } & \multicolumn{1}{|c|}{ SAT } \\
\hline & & & & & 1 & 2 \\
\hline 3 & 4 & 5 & $\begin{array}{l}6 \\
\text { HW 1 due }\end{array}$ & 7 & 8 & 9 \\
\hline 10 & 11 & $\begin{array}{l}12 \\
\text { STUDY!! }\end{array}$ & $\begin{array}{l}13 \\
\text { Quiz 1 }\end{array}$ & 14 & 15 & 16 \\
\hline
\end{tabular}




\section{When are you doing it?}

\begin{tabular}{|c|c|c|c|c|c|c|}
\hline SUN & MON & TUE & WED & THU & FRI & SAT \\
\hline & & & & & 1 & 2 \\
\hline 3 & 4 & 5 & 6 & 7 & 8 & 9 \\
\hline Study & Study & Study & HW 1 due & Study & & \\
\hline $\begin{array}{l}10 \\
\text { Study }\end{array}$ & $\begin{array}{l}11 \\
\text { Study }\end{array}$ & $\begin{array}{l}12 \\
\text { Study }\end{array}$ & $\begin{array}{l}13 \\
\text { Quiz } 1\end{array}$ & 14 & 15 & 16 \\
\hline
\end{tabular}

\section{When are you doing it?}

- Schedule your studying

- Using google calendar, other apps

Send yourself reminders before exams, HW due dates 


\section{Ask yourself:}

- Do you utilize self-testing?

- Are you taking an active role in your studying?

- Are your spacing out your studying?

\section{Specific Ways to Practice?}

- In lecture!!!

- Weekly HW

- Practice Quiz 